\title{
Frequency and Antibiotic Susceptibility of Acinetobacter Baumannii in Surgical site Infections at Rawalpindi Institute of Cardiology
}

\section{Arif Maqsood Ali ${ }^{*}$, Agha Babar Hussain ${ }^{2}$, Azhar Mehmood Kayani ${ }^{3}$}

${ }^{1}$ Consultant Microbiologist, Pathology \& Blood Bank Department, Rawalpindi Institute of Cardiology, Rawalpindi, Pakistan

${ }^{2}$ Professor \& Head of Pathology \& Blood Bank Department, Rawalpindi Institute of Cardiology, Rawalpindi, Pakistan

${ }^{3}$ Executive Director \& Professor of Cardiolgy, Rawalpindi Institute of Cardiology, Rawalpindi, Pakistan

*Corresponding author: Arif Maqsood Ali, Assistant Professor of Microbiology, Department of Pathology \& Blood Bank Rawalpindi Institute of Cardiology Rawalpindi, Punjab, Pakistan

Received date: November 28, 2019; Accepted date: December 16, 2019; Published date: January 06, 2020

Citation: Arif M. Ali., Agha B. Hussain., Azhar M. Kayani. (2020) Frequency and antibiotic susceptibility of acinetobacter Baumannii in surgical site infections at Rawalpindi Institute of cardiology. J Clinical Cardiology and Cardiovascular Interventions, 3(2); DOI:10.31579/2641-0419/031

Copyright: (C) 2020 Arif Maqsood Ali, This is an open access article distributed under the Creative Commons Attribution License, which permits unrestricted use, distribution, and reproduction in any medium, provided the original work is properly cited.

\begin{abstract}
Acinetobacter is an important nosocomial pathogen to cause infections such as pneumonia, bloodstream infections, meningitis, wound and surgical site infections and urinary tract Infections. A. baumannii can spread through direct contact with surfaces, objects and the skin of contaminated persons. Acinetobactter has acquired resistance to most of the antibiotics and can play havoc in hospital environment due to its ability to survive desiccation and in moist environment in the presence of disinfectants. Currently, there are 32 genospecies of Acinetobacter known. Among these, A. baumannii is the most important in the clinical context, since it is the most frequently isolated in nosocomial infections and the one associated with the highest mortality rate

Objective: To find out the frequency of $A$. baumannii causing surgical site infections after cardiac surgery and best antibiotic options for its treatment.

Materials and Methods: A retrospective study was carried out to find out bacterial cultures in admitted patients at Rawalpindi Institute of Cardiology with surgical site infections from July 2017 to August 2018. A total of 405 pus specimens collected from patients with suspected surgical site infections were tested for bacterial culture and antimicrobial susceptibility test. Patient profile was noted from centralized hospital data.

Results: Out of total 405 specimens 13 were culture positive for A. baumannii (3.2\%). Ten isolates were Pan Drug Resistant (PDR).

Conclusion: A. baumannii infection although infrequent in our set up is mostly pan resistant. Infection control practices \& surveillance are important to avoid its spread in hospitalized patients.
\end{abstract}

Keywords: acinetobacter baumannii; surgical site infections; coronary artery bypass graft; multi drug resistant; extended drug resistant.

\section{Introduction}

Surgical site infections (SSI) can involve any part of the body after incision and surgical procedure. It can occur in skin, organs or implanted materials. [1] It commonly occurs within 30 days after an operation under a special circumstances or within one year if an implant was placed and the infection appears to be related to the surgery. SSIs occur in $15 \%$ of all nosocomial infections among surgical patients. Post-operative SSI is the most common healthcare-associated infection in surgical patients, occurring in up to 5 percent of surgical patients. In the United States, between 500,000 to 750,000 SSIs occur annually. [2] Although Staphylococcus, Streptococcus, Pseudomonas spp. commonly cause SSIs yet nosocomial SSI due to A. baumannii following Coronary Artery Bypass Graft (CABG) is a serious and costly complication. [3]

A. baumannii is glucose-non-fermentative, non-motile, nonfastidious, catalase-positive, oxidative-negative aerobic gram-negative coccobacilli. A. baumannii is one of the most successful pathogen responsible for hospital-acquired nosocomial infections in the modern health care system. Due to the prevalence of infections and outbreaks caused by multi-drug resistant $A$. baumannii, few antibiotics are effective against it. [4]

It commonly spreads via environmental surfaces, ventilators, dialysis machine, water sources, suction equipment, as a colonizer on skin and mucosal surfaces due to its resistance to commonly used antibiotics and disinfectants which make it difficult to eradicate from hospital environment.[5]

Multi drug resistant (MDR) A. baumannii have highest resistance among countries of South East Asia although these have been reported worldwide. [6] Mortality and morbidity rates are high due to its infection. No study has been reported in SSI caused by Acinetobacter baumanii in cardiac hospitals in Pakistan.

\section{Materials and Methods}


A retrospective study was conducted from $1^{\text {st }}$ July 2017 to $31^{\text {st }}$ June 2018 in Pathology Department of Rawalpindi Institute of Cardiology (RIC), Rawalpindi Pakistan. It is a 272-bedded cardiac hospital located in Rawalpindi and provides both indoor and outdoor cardiac facilities to the patients of Rawalpindi, Islamabad and adjacent areas including patients from AJK and Northern areas.

\section{Inclusion Criteria}

All indoor and outdoor patients having SSI which were reported at RIC consecutively included in this study.

All indoor and outdoor patients having SSI who reported at RIC were included in this study.

\section{Exclusion Criteria}

Specimens collected from patients having no history of surgery were excluded. More over specimens cultured other than surgical sites were also excluded.

\section{Specimens}

Specimens from surgical sites including pus, tissues, fluids and aspirates were observed for both culture and antibiotic susceptibility.

\section{Methods of Specimen Collection}

Specimens were collected by standardized universal method before any dressing or cleaning procedure of the wound so as to avoid killing of the organism by using antiseptics. Sterile swab was taken by gently rotating it on the surgical wound site to collect exudate from the wound and was placed into transport medium. Pus aspirates were also collected by standardized method. [7]

\section{Specimen Processing}

After receiving specimen in Microbiology Laboratory, it was inoculated on blood agar, chocolate agar and MacConkey agar and culture plates were incubated at $37^{\circ} \mathrm{C}$ for 24 hours.

After receiving specimen in Microbiology Laboratory, it was processed by inoculating it on blood, chocolate and MacConkey agar and incubated plates at $37^{\circ} \mathrm{C}$ for 24 hours. The colony morphology typically showed is 1 to $2 \mathrm{~mm}$, non-pigmented, domed and mucoid colonies with smooth to pitted surfaces. [8]

Identification of gram negative rod was observed on microscopic examination by gram staining. Oxidase test was negative and confirmatory biochemical testing was done by using API 20E and NE (Biomerieux France) after incubation at $37^{\circ} \mathrm{C}$ for 24 hours. Acinetobacter does not reduce nitrate which is its distinguishing feature from Enterobacteriaceae. Indole is negative, glucose is not fermented. Antibiotic susceptibility was done by Kirby Bauer Disc Diffusion Technique and results were interpreted as per CLSI guidelines M100-S27. [8]

\section{Results}

Out of 405 pus cultures $13(3.2 \%)$ were culture positive results for $A$. baumannii whereas 180 (44.4\%) were culture negative and $212(51.4 \%)$ were positive for other isolated organisms [figure 1].
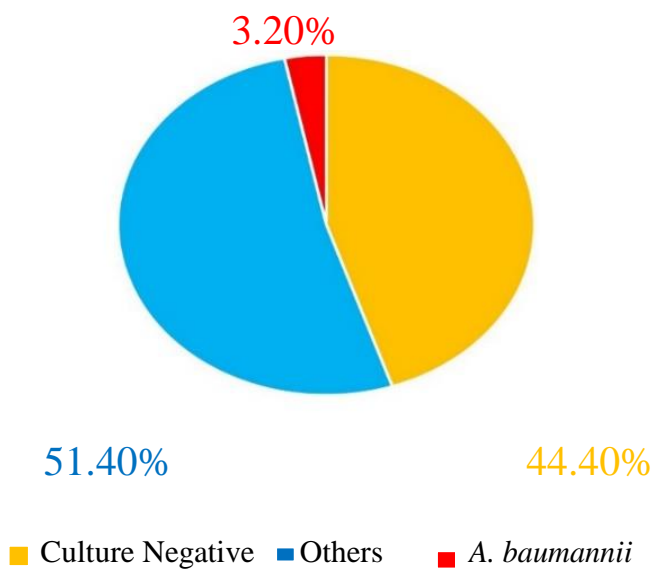

Figure 1: Frequency of A. baumannii and other isolates

Out of total 13 positive samples 7 were male patients and 6 were female patients [Figure 4].

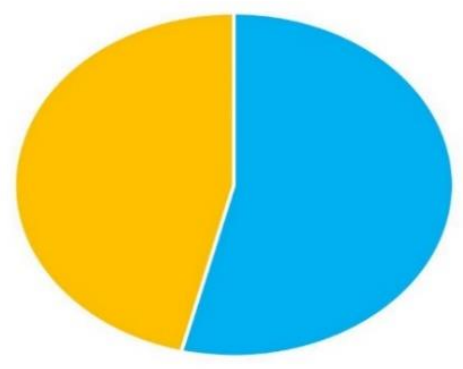

$46.10 \%$ 
- Male $\approx$ Female

Figure 2: Sex Distribution in Patients with A. baumannii

SSIs depend upon the type of surgery performed and the hospital environment. The prevalence of pathogen varies from place to place and hospital to hospital. The age distribution of all A. baumannii positive cases was above 30 and among them 8 were those having CABG while remaining 5 had different surgical procedures.

\begin{tabular}{|c|c|c|c|c|}
\hline $\begin{array}{c}\text { Serial } \\
\text { No. }\end{array}$ & Sex & Age & Surgery & Disease \\
\hline 1. & Female & 33 & $\begin{array}{l}\text { REDO- } \\
\text { MVR }\end{array}$ & Redo-MVR \\
\hline 2. & Female & 58 & $\mathrm{CABG}$ & DVCAD \\
\hline 3. & Male & 53 & $\mathrm{CABG}$ & TVCAD \\
\hline 4. & Male & 48 & CABG & TVCAD \\
\hline 5. & Male & 71 & CABG & TVCAD \\
\hline 6. & Female & 35 & BANTAL & Aortic dissection \\
\hline 7. & Male & 47 & $\begin{array}{l}\text { Wound } \\
\text { closure }\end{array}$ & Infection wound \\
\hline 8. & Female & 65 & CABG & TVCAD \\
\hline 9. & Male & 54 & $\mathrm{CABG}$ & TVCAD \\
\hline 10. & Female & 48 & CABG & TVCAD \\
\hline 11. & Female & 34 & $\begin{array}{l}\text { Wound } \\
\text { closure }\end{array}$ & Infection wound \\
\hline 12. & Male & 59 & CABG & TVCAD \\
\hline 13. & Male & 60 & $\begin{array}{l}\text { REDO- } \\
\text { MVR }\end{array}$ & REDO-MVR \\
\hline
\end{tabular}

Table 1: Profile of Infected patients

Out of 405 specimens $180(44.4 \%)$ culture negative and other culture positive isolates were Coagulase Negative Staphylococcus 70 (17.3\%), Staphylococcus aureus 68(16.8\%), Enterobacter spp. 28(6.9\%), Escherichia coli 10(2.5\%), Pseudomonas aeruginosa 10(2.5\%), Klebsiella spp. 8(1.9\%), Streptococcus spp.4(1.0\%), Enterococcus spp.4(1.0\%), Bacillus spp. and Diphtheroids 4(1.0\%), Candida spp. 4(1.0\%), Serratia spp. 2(0.5\%)[Figure 5]. 


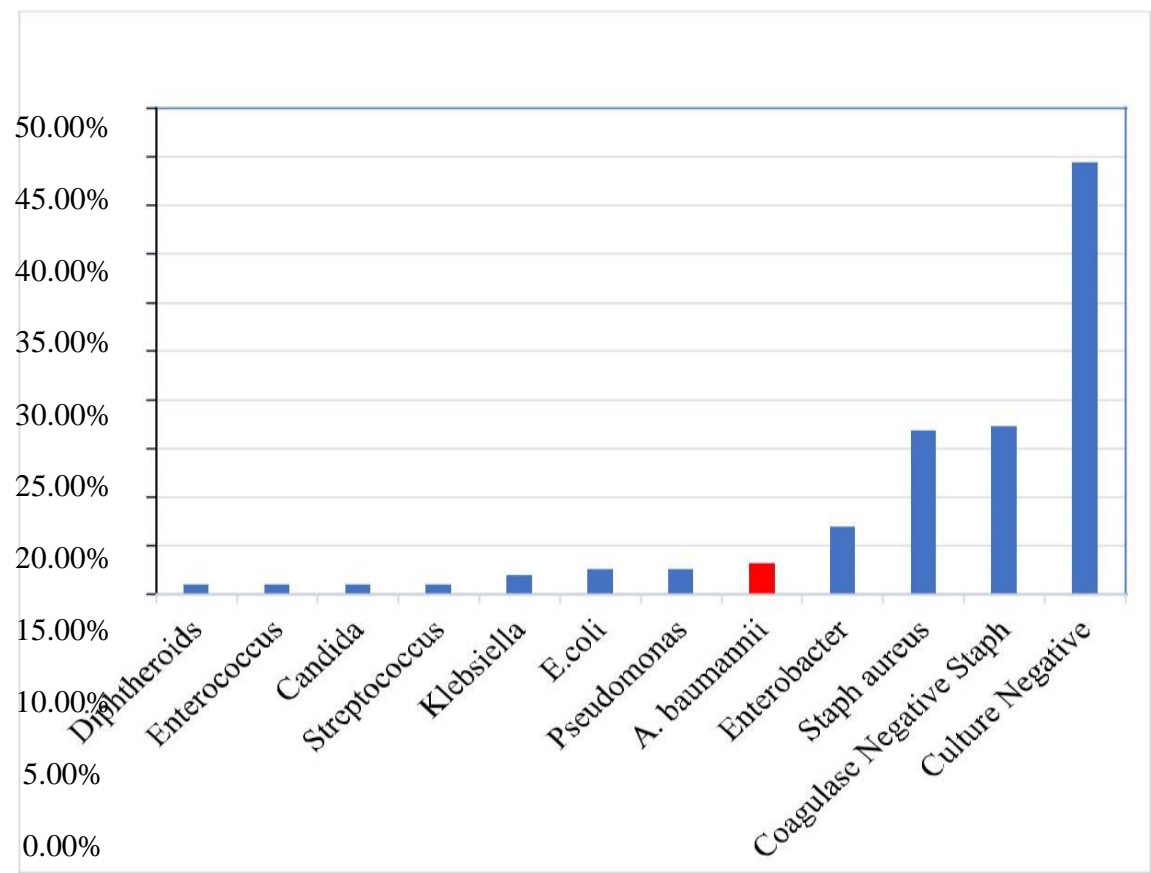

Figure 3: Frequency of bacterial species isolated from clinical specimens

Out of 13 positive A. baumannii isolates 10 isolates were pan resistant and 3 isolates showed partial susceptibility to antibiotics. Among these, the first isolate was sensitive to Amikacin, Gentamycin, Ciprofloxacin, Chloramphenicol, Tazobactam/Piperacillin, the Second isolate to Levofloxacin, Sulphamethoxazole, Meropenem, Imipenem, Ciprofloxacin and the third isolate to Meropenem, Imipenem, Amikacin and Gentamycin.

\begin{tabular}{|l|l|l|l|l|}
\hline \multicolumn{1}{|c|}{ Antibiotics } & Sensitive Out of 13 & Sensitivity \% & Resistant Out of 13 & Resistance \% \\
\hline Amikacin & 02 & $15.3 \%$ & 11 & $84.6 \%$ \\
\hline Augmentin & 0 & $0 \%$ & 13 & $100 \%$ \\
\hline Gentamycin & 02 & $15.3 \%$ & 11 & $84.6 \%$ \\
\hline Aztreonam & 0 & $0 \%$ & 13 & $100 \%$ \\
\hline Cefixime & 0 & $0 \%$ & 13 & $100 \%$ \\
\hline Ceftriaxone & 0 & $0 \%$ & 13 & $100 \%$ \\
\hline Piperacillin Tazobactam & 01 & $7.7 \%$ & 12 & $92.3 \%$ \\
\hline Imipenem & 02 & $15.3 \%$ & 11 & $84.6 \%$ \\
\hline Meropenem & 02 & $15.3 \%$ & 11 & $84.6 \%$ \\
\hline Ciprofloxacin & 02 & $15.3 \%$ & 11 & $84.6 \%$ \\
\hline Levofloxacin & 01 & $7.7 \%$ & 12 & $92.3 \%$ \\
\hline Sulphamethoxazole & 01 & $7.7 \%$ & 12 & $92.3 \%$ \\
\hline Tigecycline & 0 & $0 \%$ & 13 & $100 \%$ \\
\hline Doxycycline & 0 & $0 \%$ & 13 & \\
\hline
\end{tabular}




\begin{tabular}{|l|l|l|l|l|} 
Polymyxins & 0 & $0 \%$ & 13 & $100 \%$ \\
\hline
\end{tabular}

Table 2: Antibiotic susceptibility Pattern

\section{Discussion}

A. baumannii has emerged as the causative agent of nosocomial infection due to increased use of invasive procedures, overuse of broad spectrum antimicrobials and prolonged duration of stay in the hospital. Development of resistance against antimicrobials is a major problem in the treatment of Acinetobacter infections. Although they are considered as pathogen of mild virulence, they can rapidly acquire resistance. [9]

A. baumannii is an opportunistic pathogen. It has a high incidence among immunocompromised individuals, particularly those who have experienced a prolongedHospital stay. [10] It may colonize the skin as well as being isolated in high numbers from the respiratory, surgical and oropharynx secretions of infected individuals. [11] In recent years, it has been designated as a "red alert" human pathogen, generating alarm among the medical community, arising largely from its extensive antibiotic resistance spectrum. [12]

SSIs are one of the common post-operative complications. Apart from bacterial contamination of wound, various patient and environment related factors play role in development and outcome of SSIs. The present study is undertaken to study the frequency of of A. baumannii SSIs after cardiac surgery and its antimicrobial susceptibility. The prevalence of pathogen varies from place to place and hospital to hospital. [13]

Most of the isolates were considered as highly resistant to Imipenem, Amikacin, and Ampicillin/Sulbactam. MDR Acinetobacter spp. is defined as those isolates resistant to more than three classes of antibiotics (Penicillin's and Cephalosporins, Fluoroquinolones and Aminoglycosides). An isolate is classified as extended drug resistant (XDR) when it is resistant to three classes of antimicrobials described above (MDR) and is also resistant to Carbapenems. Pan drug resistant isolate define as that is resistant to Polymyxins and Tigecycline. [14]

CDC considers A. baumannii is a serious public health hazard due to its resistance to multiple antibiotics. [15]

SSI are still common in patients despite great advances in surgical techniques such as prophylactic antibiotics, disinfection and sterilization. Numerous studies have reported nosocomial infections after cardiac surgery to exceed $20 \%$. The incidence of nosocomial infections was reported as $16 \%$ in the study by (Lomtadze et al) and $8.3 \%$ in the study by (Davoodi et al). [16]

Microbiological evidence suggests that $5 \%$ of open heart surgery patients develop nosocomial infections. The health authorities need to take measures at the ministerial and regional levels in order to reduce this under-reporting. [16]

A. baumannii has emerged as an important pathogen affecting any age group but in our study, it is most commonly found in patients between 3070 years. In a previous study the most common age group effected by $A$. baumannii was between $42-68$ years. [17] It is probably due to early onset of IHD in patients requiring Cardiac surgery.

A. baumannii was reported in surgical wound infection in Turkey and Saudi Arabia in $8.3 \%$ and $8.6 \%$ cases respectively. In another study reported in Saudi Arabia, the frequency of A. baumannii was $22.2 \%$ in wound infections. [18]

A. baumannii Infections were frequently isolated in males (53.8\%) than females $(46.10 \%)$ in our study. It is similar to results reported in a study from Saudi Arabia where out of 131 patients operated, 93 (71\%) were males. The higher number of males may be due to the fact that males are more prone to develop cardiovascular diseases than females that require surgery [19].

A. baumannii infections are associated with major risk factors such as post-surgical, catheterization, extended hospital stays and prolonged antibiotic usage. A. baumannii isolated from normal skin and mucous membranes are reported to cause serious and sometimes fatal infections [20].

Since, our study is based in a cardiac set up A. baumannii infections were found in cardiac patients having diseases like Triple or Double Vessel Coronary Artery Disease (TVCAD) or (DVCAD), Redo-Mitral Valve Replacement Surgery (Redo- MVR), aortic dissection and open-heart surgery. Infection occurred in patients who were admitted to ICU for stabilization after a surgical procedure and required mechanical ventilation.

Infectious complications after cardiac surgery occur in $5 \%$ to $21 \%$ of cases. [21] Major infectious complications increase postoperative mortality by more than 5 times and prolong recovery. [22] Forty seven percent of these patients require more than 14 days in the hospital compared with $5.9 \%$ of patients without a major infection. [23] As a result, infectious complications often increase the cost of care. [24] However, infectious complications can be reduced with many simple interventions, by risk factor modification preoperatively to postoperative risk factor vigilance in the ICU.

In a review, the most common sites of infection reported were respiratory tract $(45.7 \%-57.8 \%)$, surgical site $(27.7 \%)$, and catheters or devices $(20.5 \%-25.2 \%)$ occurring perioperatively or within the first 12 months of surgery. [25]

In our study out of 405 specimens commonly isolated culture positive organisms are Coagulase Negative Staphylococcus 70 (17.3\%), Staphylococcus aureus 68 (16.8\%), Enterobacter spp. 28 (6.9\%), Escherichia.coli 10 (2.5\%), Pseudomonas aeruginosa 10 (2.5\%), Klebsiella spp. 8 (1.9\%), Streptococcus spp. 4 (1.0\%), Enterococcus spp. $4(1.0 \%)$, Bacillus spp. and Diphtheroids $4(1.0 \%)$, Candida spp. 4 (1.0\%), Serratia spp. 2 (0.5\%) along with A. baumannii (3.2\%) (Fig. 3). Similar to our study a bacteriological study of SSIs carried out in a tertiary care hospital at India concluded that overall frequency of SSI was $6.17 \%$. Most common isolates were Staphylococcus aureus, Coagulase negative Staphylococci (CONS), E. coli and Pseudomonas aeruginosa. The frequency of Methicillin Resistant Staphylococcus aureus (MRSA) was $8.6 \%$. The maximum frequency was among patients operated on emergency basis in surgical department. [26]

A. baumannii cause skin or soft tissue infections outside the military population leading to $2.1 \%$ of ICU-acquired skin or soft tissue infections. A. baumannii was isolated from soldiers with open tibia fractures in Iraq or Afghanistan war. [27]

A. baumannii is a nosocomial pathogen and due to its resistance pattern, it is one of the leading cause of SSIs in our setup. Antibiotic susceptibility pattern of Acinetobacter spp. against various antibiotics in the present study showed (15.3\%) susceptibility to Meropenem, Imipenem, Gentamicin, Ciprofloxacin \& Amikacin whereas for Piperacillin/Tazobactam combination, Levofloxacin and Trimethoprim/ Sulphamethoxazole it was (7.7\%). All isolated were resistant to Augmentin, Aztreonam, Cefixime, Ceftriaxone, Tigecycline, Doxycycline and Polymyxins (Table 2). A study from India reported antibiotic susceptibility of Acinetobacter species to Meropenem (86\%), Piperacillin Tazobactam (76\%), Amikacin (67\%), Ampicillin/ Sulbactam $(58 \%)$, Gentamicin (50\%), Ciprofloxacin (49\%), Ceftazidime (40\%), Tetracycline $(31 \%)$, Cotrimoxazole $(29 \%)$, Ceftriaxone $(21 \%)$ and Cefepime $(18 \%){ }^{28}$

In another Study $52.5 \%$ cultures yielded significant growth and only $3 \%$ Acinetobacter spp. were isolated. Maximum (56.9\%) isolates were obtained from pus/swab, followed by blood (13.1\%) and urine $(12.4 \%)$. Elderly age, being inpatients, longer duration of stay in the hospital, associated co-morbidity, and invasive procedure were found to be significant risk factors in the setup investigated. Out of $3 \%$ A. baumannii isolates, $54.7 \%$ were resistant to more than three classes of antibiotics 
MDR and 5.8\% were resistant to all commonly used antibiotics PDR. Majority of the isolates were sensitive to Imipenem, Meropenem, and Piperacillin/Tazobactam, and showed resistance rates of 19\%, 22\%, and $23 \%$, respectively. ${ }^{29}$

In a study from Brazil where in a six-month period, 1333 patients were admitted to the five ICUs included. The mean age of the participants was 53.2 years, and $59.4 \%$ were male. Most patients (98.4\%) had previously taken antimicrobials and $68.2 \%$ had used two or more classes of antimicrobial drugs. The most commonly used drugs were $3^{\text {rd-and }} 4^{\text {th }}$ generation Cephalosporins (71.4\%) and Carbapenems (50.8\%). [30]

Another study at Pakistan reveals that isolation rate of Acinetobacter was high from the respiratory specimens, followed by wound samples. Antibiotic susceptibility of the isolates revealed that the resistance to Cefotaxime and Ceftazidime was the most common, in $710(99.2 \%)$ specimens each, followed by the resistance to Gentamicin in $670(93.6 \%)$ isolates, and to Imipenem in $651(90.9 \%)$ isolates. However, almost all isolates were susceptible to Tigecycline, Colistin, and Polymyxins B. [31] In an international study in ICUs, the Acinetobacter infection rate was $19.2 \%$ in Asia, $17.1 \%$ in Eastern Europe, $14.8 \%$ in Africa, $13.8 \%$ in Central and South America, 5.6\% in Western Europe, $4.4 \%$ in Oceania and $3.7 \%$ in North America [32].

Global data reveals that multidrug-resistant $A$. baumannii is emerging as

\section{References}

1. Hemant Singhal (2018), Wound Infection Clinical Presentation Kitembo, S. K., \& Chugulu, S. G. (2013). Incidence of surgical site infections and microbial pattern at Kilimanjaro Christian medical centre. Annals of African Surgery, 10(1). Surgical Site Infections | Johns Hopkins Medicine Health Library

2. Lee, C. R., Lee, J. H., Park, M., Park, K. S., Bae, I. K., Kim, Y. B., Cha, C. J., Jeong, C., Lee, S. H. (2017). Biology of Acinetobacter baumannii: Pathogenesis, Antibiotic Resistance Mechanisms, and Prospective Treatment Options. Frontiers in cellular and infection microbiology, 7, 55. Acinetobacter in Healthcare Setting CDC - Nov 24, 2010

3. Hsu, L. Y., Apisarnthanarak, A., Khan, E., Suwantarat, N., Ghafur, A., \&Tambyah, P. (2017). Carbapenem-resistant Acinetobacter baumannii and Enterobacteriaceae in south and southeast Asia. Clinical microbiology reviews, 30(1), 1-22.

4. Pus, surgical specimens and tissue Pathology Identification of Acinetobacter species. NCB-NIH

5. Alsan, M., \& Klompas, M. (2010). Acinetobacter baumannii: an emerging and important pathogen. Journal of clinical outcomes management: JCOM, 17(8), 363.

6. Montefour K, Frieden J, Hurst S, Helmich C, Headley D, Martin M, et al. Acinetobacter baumannii: an emerging multidrug-resistant pathogen in critical care. Crit Care Nurse. 2008; 28:15-25, quiz 26.

7. Sebeny PJ, Riddle MS, Petersen K. Acinetobacter baumannii skin and soft-tissue infection associated with war trauma. Clinical Infectious Disease. 2008; 47: 444-449.

8. Cerqueira GM, Peleg AY. Insights into Acinetobacter baumannii pathogenicity. IUBMB Life. 2011; 63: 1055-1060.

9. Lomtadze M, Chkhaidze M, Mgeladze E, Metreveli I, Tsintsadze A. Incidence and risk factors of nosocomial infections after cardiac surgery in Georgian population with congenital heart diseases. Georgian Med News. 2010 Jan; 178:7-11.

10. Manian, F. A. (2014). The role of postoperative factors in surgical site infections: time to take notice. Clinical Infectious Diseases, 59(9), 1272-1276.

11. Falagas, M. E., Koletsi, P. K., \&Bliziotis, I. A. (2006) The diversity of definitions of Multidrug-Drug Resistant (MDR) and Pan Drug-Resistant (PDR) Acinetobacter baumannii and a common hospital-and community-acquired infection that is difficult to treat. It is a very resistant and aggressive organism that infects patients with weakened defences like ICU patients and those with invasive devices. [33]

It is important to notify multi drug resistant $A$. baumannii (MDR-Ab). For patients colonized or infected with MDR-Ab maximum isolation precautions require a private room and one to one nursing. Each patient with MDR-Ab needs dedicated equipment and universal precautions should be followed to attend patients with MDR-Ab.

\section{Conclusion}

The frequency of A. baumannii infections in our study is $3.20 \%$ in surgical site infections. SSI is more common in males $(53.80 \%)$ than females $(46.20 \%)$. Most of these isolates were pan resistant. In order to decrease the spread of $A$. baumannii infections and reduce its resistance, it is important to promote the rational use of antimicrobials, with implementation and monitoring of the Antibiotics Stewardship Program in hospitals. Hand hygiene and barrier nursing are important to prevent its spread.

Pseudomonas aeruginosa. Journal of medical microbiology, 55(12), 1619-1629.

12. Manchanda, V., Sanchaita, S., \& Singh, N. (2010). Multidrug resistant acinetobacter. Journal of global infectious diseases, 2(3), 291-304.

13. Heydarpour, F., Rahmani, Y., Heydarpour, B., \& Asadmobini, A. (2017). Nosocomial infections and antibiotic resistance pattern in open-heart surgery patients at Imam Ali Hospital in Kermanshah, Iran. GMS hygiene and infection control, 12.

14. Uwingabiye, J., Frikh, M., Lemnouer, A., Bssaibis, F., Belefquih, B., Maleb, A., \&Louzi, L. (2016). Acinetobacter infections prevalence and frequency of the antibiotics resistance: comparative study of intensive care units versus other hospital units. Pan African Medical Journal, 23(1).

15. Al-Gethamy, M. M., Faidah, H. S., Adetunji, H. A., Haseeb, A., Ashgar, S. S. et al. (2017). Risk factors associated with multidrug-resistant Acinetobacter baumannii nosocomial infections at a tertiary care hospital in Makkah, Saudi Arabia - a matched case-control study. The Journal of international medical research, 45(3), 1181-1189.

16. Khairy, G. A., Kambal, A. M., Al-Dohayan, A. A., Al-Shehri, M. Y., Zubaidi. et al. (2011). Surgical site infection in a teaching hospital: a prospective study. Journal of Taibah University Medical Sciences, 6(2), 114-120.

17. Tripathi, P. C., Gajbhiye, S. R., \& Agrawal, G. N. (2014). Clinical and antimicrobial profile of Acinetobacter spp.: An emerging nosocomial superbug. Advanced biomedical research, 3,13 .

18. Kollef MH, Sharpless L, Vlasnik J, et al. (1997) The impact of nosocomial infections on patient outcomes following cardiac surgery. Chest. 112: 666-675.

19. Michalopoulos A, Geroulanos S, Rosmarakis ES, et al. (2006) Frequency, characteristics, and predictors of microbiologically documented nosocomial infections after cardiac surgery. Europian Journal Cardiothorac Surgery. 29: 456-460.

20. Fowler VGJ, O'Brien SM, Muhlbaier LH, et al. (2005) Clinical predictors of major infections after cardiac surgery. Circulation. 112: I358-I365.

21. Jenney AW, Harrington GA, Russo PL, et al. (2001) Cost of surgical site infections following coronary artery bypass surgery. ANZ J Surg. 71:662-664. 
J Clinical Cardiology and Cardiovascular Interventions

22. Cove, M. E., Spelman, D. W., \&MacLaren, G. (2012). Infectious complications of cardiac surgery: a clinical review. Journal of cardiothoracic and vascular anesthesia, 26(6), 1094-1100.

23. Bhave, P. P., Kartikeyan, S., Ramteerthakar, M. N., \& Patil, N. R. (2017). Bacteriological study of surgical site infections in a tertiary care hospital at Miraj, Maharashtra state, India. International Journal of Research in Medical Sciences, 4(7), 2630-2635.

24. Peleg, A. Y., Seifert, H., \& Paterson, D. L. (2008). Acinetobacter baumannii: emergence of a successful pathogen. Clinical microbiology reviews, 21(3), 538-582.

25. Ranganath, R., \& Vijaykumar, G. S. (2016). Antimicrobial Sensitivity Pattern of Acinetobacter Species Isolated from Clinical Specimens. Int. J. Curr. Microbiol. App. Sci, 5(12), 519523.

26. Dash, M., Padhi, S., Pattnaik, S., Mohanty, I., \& Misra, P. (2013). Frequency, risk factors, and antibiogram of Acinetobacter species isolated from various clinical samples in a tertiary care hospital in Odisha, India. Avicenna journal of medicine, 3(4), 97-102.
27. Castilho SRA, Godoy CSdM, Guilarde AO, Cardoso JL, André MCP, Junqueira-Kipnis AP, et al. (2017) Acinetobacter baumannii strains isolated from patients in intensive care units in Goiânia, Brazil: Molecular and drug susceptibility profiles. PLoS ONE 12(5): e0176790.

28. Sohail, M., Rashid, A., Aslam, B., Waseem, M., Shahid, M., Akram, M., \& Rasool, M. H. (2016). Antimicrobial susceptibility of Acinetobacter clinical isolates and emerging antibiogram trends for nosocomial infection management. Revista da Sociedade Brasileira de Medicina Tropical, 49(3), 300-304.

29. Vincent JL, Rello J, Marshall J, Silva E, Anzueto A. (2009) EPIC II Group of Investigators. International Study of the Prevalence and Outcomes of Infection in Intensive Care Units. JAMA. 302(21):2323-2329.

30. Begum, S., Hasan, F., Hussain, S., \& Ali Shah, A. (2013). Prevalence of multi drug resistant Acinetobacter baumannii in the clinical samples from Tertiary Care Hospital in Islamabad, Pakistan. Pakistan journal of medical sciences, 29(5), 12531258.

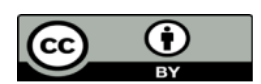

This work is licensed under Creative Commons Attribution 4.0 License

\section{To Submit Your Article Click Here: Submit Article}

DOI: $10.31579 / 2641-0419 / 031$
Ready to submit your research? Choose Auctores and benefit from:

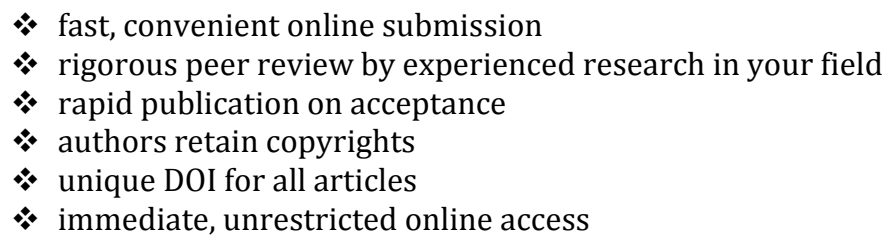

At Auctores, research is always in progress.

Learn more www.auctoresonline.org/journals/clinical-cardiology-andcardiovascular-interventions 